\title{
Traduire
}

Revue française de la traduction

234 | 2016

La traduction, un sport de haut niveau

\section{Le cauchemar des interprètes}

\section{Laurent Laget}

\section{(2) OpenEdition \\ Journals}

Édition électronique

URL : http://journals.openedition.org/traduire/790

DOI : 10.4000/traduire.790

ISSN : 2272-9992

Éditeur

Société française des traducteurs

Édition imprimée

Date de publication : 15 juin 2016

Pagination : 18-23

ISSN : 0395-773X

\section{Référence électronique}

Laurent Laget, « Le cauchemar des interprètes », Traduire [En ligne], 234 | 2016, mis en ligne le 15 juin

2018, consulté le 07 mai 2019. URL : http://journals.openedition.org/traduire/790 ; DOI : 10.4000/

traduire.790 


\section{Le cauchemar des interprètes}

\section{Laurent Laget}

Je suis un gosse de la Génération 84, celle de l'équipe de France championne d'Europe. De I'Italie championne du monde deux ans plus tôt. Au point que ma famille, franco-italienne, m'a surnommé « bébé-Platini " lorsque j'ai tapé dans mon premier ballon, avant même de pouvoir mettre un pied devant l'autre.

C'est peut-être de là que vient ma fascination pour les joueurs de football. Pas pour leurs voitures et leurs maisons aussi démesurées que leur ego, ni même pour leur talent balle au pied, à quelques exceptions près.

J'ai grandi avec le beau Paris Saint-Germain, celui des Rai, Ginola, Weah et Leonardo. Et ce qui éblouissait mes yeux d'ado, c'était avant tout leurs talents linguistiques. Prenons le Brésilien Leonardo. Né dans la langue de Camões, il débarque en Espagne où il n'a aucun mal à apprendre la langue de Cervantes. II s'initie ensuite à celle de Mishima pendant deux ans dans le club de Kashima, avant de découvrir la capitale française et la langue de Molière. Leonardo continue son périple à Milan, où Dante n'a pas longtemps gardé ses secrets. Pendant tout ce temps, il peaufine son Shakespeare footballistique. Six langues, des voyages dans le monde entier, une sensibilité interculturelle affûtée. Il y a de quoi faire rêver, avouez.

Puis vint José Mourinho. L'entraîneur à la fois le plus adulé et le plus détesté de notre époque n'a pas connu une grande carrière de joueur, contrairement à nombre de ses homologues. Dans une autre vie, José Mourinho était interprète. II fait ses premières armes aux côtés de l'Anglais Sir Bobby Robson, alors coach du Sporting de Lisbonne, pour l'aider à communiquer avec les lusophones de l'équipe. II suit alors son mentor à Barcelone, où il apprend le catalan. En parallèle, Mourinho acquiert des rudiments de français et d'italien. Et hop, six langues de travail dans sa besace. Un bagage qui lui vaudra d'être surnommé, bien malgré lui, "le traducteur ". À ses débuts, Mourinho se heurte pourtant de plein fouet à la réalité qui touche notre profession, puisque le jeune adjoint-interprète touche la modique somme de 10000 pesetas par mois (l'équivalent de 60 euros !) pour ses services linguistiques en Catalogne. Lucide, il 
comprend rapidement l'intérêt de la diversification et offre des services de conseils tactiques qui s'avéreront bien plus lucratifs.

Aujourd'hui, c'est lui qui est traduit dans toutes les langues. Et autant dire qu'il veille au grain. Mourinho est devenu au fil des ans la terreur des interprètes en conférence de presse. L'autoproclamé Special One a bâti sa légende autant sur ses résultats que sur son franc-parler.

Ainsi, dès qu'un interprète ose s'égarer dans sa formulation, vous pouvez être sûr que Mourinho lui tombera dessus. Ambiance.

\section{Acte I}

Septembre 2010. De passage à Auxerre avec son équipe du Real Madrid, Mourinho se voit demander si son équipe méritait de gagner ce soir-là.

Mourinho: We had some matches in Spain where we had 15 chances to score. We didn't score. We draw 0-0 against Mallorca. We draw 0-0 against Levante. And everybody was saying we didn't deserve to win because we didn't score. Today we scored, so today we deserve to win(1).

L'interprète : D'après l'entraîneur, nous méritons de gagner, malgré les matchs nuls contre Levante et Majorque.

Mourinho : Non. [Hilarité générale]

L'interprète : Non?

Mourinho : J'ai dit que contre Levante et Majorque, nous avons créé 15 situations de but, nous n'avons pas marqué, les matchs se sont terminés 0-0 et tout le monde a dit que nous ne méritions pas de gagner le match. Aujourd'hui 1-0. Si tout le monde est pragmatique, OK, nous méritons de gagner le match.

\section{Acte II}

Décembre 2011. Le Real de Mourinho voyage à Amsterdam, sur les terres de l'Ajax. La conférence d'avant-match se déroule en anglais.

(1) Nous avons joué certains matchs en Espagne pendant lesquels nous nous sommes procuré 15 occasions de but, mais nous n'avons pas marqué. Nous avons fait 0-0 contre Majorque. Nous avons fait 0-0 contre Levante. Et tout le monde a dit que nous ne méritions pas de gagner parce que nous n'avons pas marqué. Aujourd'hui, on a marqué, alors on mérite de gagner. 
Mourinho : I thought the history of my punishment would be a start for a better football. And if I have to be punished for a better football, I would be pleased to do that. But no, it was just for me. Football is the same, people do the same, people are not punished, so it was just for me(2).

L'interprète : Siempre es un buen sentido estar otra vez aquí en Ámsterdam. Lo que pasó puede pasar a cualquier persona y miramos al futuro(3).

Mourinho : No. Lo siento, no. No he dicho esto. Lo siento. Si no lo entiende, no tengo que repetir. No soy traductor(4).

\section{Acte III}

Décembre 2013. Souvent mauvais perdant, Mourinho peut aussi être beau joueur. (Re)devenu entraîneur du club londonien de Chelsea, son équipe se déplace en Roumanie. Après une longue tirade du Portugais, l'interprète roumain passe plusieurs minutes à restituer ses propos. Si on peut légitimement penser que le Portugais n'est cette fois pas apte à juger de la qualité de la traduction, il en reconnaît toutefois le mérite. C'est ainsi que Mourinho lance : "Qui paye ce type ? II faut lui doubler son salaire ! ", avant de lui remettre son sweatshirt dédicacé en fin de conférence.

\section{Acte IV}

Octobre 2014. Notre Raymond Domenech national, qui n'a pas non plus sa langue dans sa poche, publie son abécédaire du football. Pas franchement copain avec son homologue portugais, il lui dédicace ce petit mot doux : "C'est le problème des traducteurs, ils finissent par se persuader d'avoir écrit le texte eux-mêmes". (Désolé Raymond, nous sommes bien les auteurs de nos textes traduits, je serai ravi de te faire parvenir une copie du code de propriété intellectuelle !)

(2) J'ai cru que ma sanction serait le point de départ d'un football meilleur. Et si je dois être puni pour que le football s'améliore, je l'accepte volontiers. Mais non, c'était personnel. Le football reste le même, les gens agissent toujours de la même manière et ne sont pas punis. Ce n'était que pour moi.

(3) C'est toujours agréable de revenir ici à Amsterdam. Ce qu'il s'est passé aurait pu arriver à n'importe qui, et nous sommes tournés vers l'avenir.

(4) Non. Désolé, mais non, je n’ai pas dit ça. Je suis désolé. Si vous n'avez pas compris, je ne vais pas répéter. Je ne suis pas traducteur. 
Ce à quoi Mourinho répondra avec une grande justesse :

I think because I can speak five languages, it doesn't make me a translator. That makes me an awful translator because I am not very, very good in any one of these languages. I was just trying to help my boss - the manager at that time - to communicate the best way with the players and the media. Don't call me a translator because that would be an offence to every translator. But it helps me, the fact that I can communicate(5).

Outre ses saillies oratoires, Mourinho s'est avant tout distingué par ses qualités managériales, son sens de la tactique et les titres qu'il a obtenus avec ses équipes successives. Comme il le dit lui-même, sa maîtrise des langues à des fins professionnelles l'a aidé à mieux communiquer. Ce n'est pas négligeable lorsque vous devez diriger un groupe de gamins venus du Brésil, de France, de Suède et de Croatie !

Dès lors, doit-on impérativement maîtriser plusieurs langues pour faire carrière dans le football ? II est certes possible de gravir les échelons en restant dans son pays, mais les plus grandes figures du foot ont souvent baroudé dans les cinq grands championnats (Allemagne, Angleterre, Espagne, France, Italie) pour asseoir leur notoriété. Les exceptions existent, à l'instar de Lionel Messi : l'Argentin n'a connu que Barcelone, mais n'en est pas moins considéré comme le meilleur joueur de l'histoire.

II faut dire que les clubs de football disposent de ressources financières et humaines souvent colossales : ainsi, lorsqu'un joueur ou un entraîneur étranger débarque dans une nouvelle équipe, il n'est pas rare que son employeur lui impose des cours intensifs de langue pour mieux l'acclimater à son nouvel environnement. Tout dépend de la configuration du vestiaire : une équipe homogène, constituée de joueurs d'une même nationalité mais dirigée par un entrâ̂neur étranger, se contentera d'un interprète, comme ce fut le cas pour Mourinho. À l'inverse, dans une équipe bigarrée les individualités (linguistiques) peuvent faciliter l'intégration.

À ce titre, le Paris Saint-Germain constitue un cas d'école. Racheté en 2011 par de grandes fortunes qataries, le PSG a changé de dimension financière et sportive du jour au lendemain, attirant subitement de grands joueurs de tous horizons, notamment italiens et brésiliens (italophones pour la plupart). Salvatore Sirigu est l'exemple parfait d'une intégration réussie : fraîchement débarqué de Sicile, l'humble gardien de but n'a mis que quelques semaines pour maîtriser le français et se faire le porte-parole du vestiaire.

Certes, on pourrait estimer que c'est bien la moindre des choses d'apprendre la langue du pays dans lequel on vit et travaille. Mais n'oublions pas que ces sportifs évoluent dans une bulle

(5) Ce n'est pas parce que je parle cinq langues que cela fait de moi un traducteur. Du moins, cela fait de moi un très mauvais traducteur, parce que je n'excelle dans aucune de ces langues. J'essayais juste d'aider mon patron, l'entraîneur à l'époque, à communiquer du mieux possible avec les joueurs et les médias. Ne me qualifiez pas de traducteur, ce serait une insulte à tous les traducteurs. Mais le fait que je puisse communiquer ainsi m'aide dans mon travail. 
dorée. Ainsi, il n'est pas impensable qu'un footballeur ne s'intéresse qu'à ses performances et son porte-monnaie, et non à la découverte culturelle et linguistique. Toujours au PSG, Zlatan Ibrahimovic est sans doute l'antithèse de Sirigu. Après trois ans en France, il aura fallu que l'égocentrique suédois soit interviewé en italien pour l'entendre admettre qu'il « ne comprend pas le français ".

Les exemples et contre-exemples sont innombrables : Frank Ribéry, admiré pour ses expressions colorées (et sa fameuse " la routourne va tourner "), parle aujourd'hui un allemand compréhensible après plusieurs années en Bavière. L'Uruguayen Diego Forlan, quant à lui, n'a pas hésité à se mettre au japonais lors de sa pige à Osaka. Le Sénégalais Babacar Diallo, parti faire ses armes à Istanbul, affirme avoir pu apprendre un peu le turc (même si " leur clavier pour les ordinateurs est totalement différent du nôtre. Tu as des i tréma partout ! ") avant de partir en Finlande où il est au contraire resté réfractaire au finnois.

On peut donc en conclure que si la maîtrise des langues étrangères ne paraît pas de prime abord indispensable pour ces acteurs du football qui évoluent en vase clos, il est indéniable que les langues constituent, comme partout, un puissant vecteur d'intégration. Et il est bien connu qu'une acclimatation réussie favorise le bien-être et, par conséquent, les performances sportives.

Je ne peux conclure ce billet sans relater une anecdote juteuse sur l'Olympique de Marseille, qui a récemment connu quelques déboires avec ses interprètes (qui se présente ivre, qui traduit à côté de la plaque, qui se spécialise dans les néologismes de son cru). En déplacement à Guingamp, l'encadrement de l'OM se retrouve dépourvu d'interprète pour la conférence de presse de l'entraîneur argentin Marcelo Bielsa. L'hôtel où séjourne le groupe fait des pieds et des mains et finit par trouver un pompier de service à la dernière minute : c'est ainsi que le directeur d'un supermarché local se retrouva propulsé interprète. Un futur Mourinho ?

Quant à Mourinho, justement, que peut-on retenir de son expérience ? Spécialisez-vous pour trouver des missions, et si vous n'êtes pas satisfaits de votre rémunération, diversifiez votre offre de services!

laurent@anothertranslator.eu

Laurent Laget est traducteur indépendant de l'anglais et de l'italien depuis 2008. Son diplôme de l'ESIT en poche, il cherche sa voie en travaillant pour des institutions internationales et des agences quand il découvre le monde de la traduction sportive. Ce "sportif de clavier" se fait happer par les ballons ronds et ovales, les petites reines et les grands fonds. Le monde de l'édition s'ouvre à lui et il élargit ses compétences aux domaines de la santé et de l'alimentation. Laurent est membre de la Société française des traducteurs et de l'Association des traducteurs littéraires de France. II vit à Paris avec sa femme, son fils et ses deux chats. 


\section{Sources}

PEREIRA William, 30 septembre 2014, Jose Mourinho, "le traducteur ", Sofoot.com, http://www.sofoot.com/jose-mourinho-le-traducteur-189725.html

LAMBERT Maxime, 9 février 2011, FC Barcelone : Quand José Mourinho gagnait 60 euros par mois, Gentside Sport, http://sport.gentside.com/fc-barcelone/

fc-barcelone-quand-jose-mourinho-gagnait-60-euros-par-mois art10925.html

DUCHÂTEAU Romain, 28 août 2015, Babacar Diallo : "Le finnois est presque la troisième langue en Finlande ", Sofoot.com, http://www.sofoot.com/babacar-diallo-le-finnois-est-presquela-troisieme-langue-en-finlande-207156.html

Le Scan Sports, 26 octobre 2015, Ibrahimovic ne parle pas aux médias parce qu'il " ne comprend pas le français ", Le Figaro.fr, http://sport24.lefigaro.fr/le-scan-sport/ medias/2015/10/26/27005-20151026ARTFIG00152-ibrahimovic-ne-parle-pas-aux-mediasfrancais-parce-qu-il-ne-comprend-pas-le-francais.php

L'équipe.fr, 1er octobre 2015, L'OM et ses interprètes, toute une histoire, http://www.lequipe.fr/Football/Actualites/L-om-et-ses-interpretes-toute-une-histoire/595001 DOMENECH Raymond, 2014, Mon dico passionné du foot, Paris, Flammarion. 